\title{
A mathematical model of the Multiverse described by exploded numbers
}

\author{
Szalay, I. \\ Department of Mathematics, University of Szeged, Hungary \\ Email: szalay.istvan22@gmail.com \\ Corresponding author: Szalay
}

\begin{abstract}
.
The theory of exploded and compressed numbers is a relatively unknown area of Mathematics. By the compressed numbers we are able to compress our Universe into an open cube. On the other hand using the exploded numbers, by the explosion our Universe becomes Multiverse which contains infinitely many three dimensional spaces such that among them one is the our Universe. We show a certain continuation of an unbounded set of our Universe such that this continuation is situated in another universe. Moreover, we present some passages of the way of a surveyor crossing parallel universes.
\end{abstract}

Keywords: compression, explosion, prallel Universes, Multiverse

\section{INTRODUCTION}

Let $x$ be an arbitrary real number. The ordered pair (0.1)

$((\operatorname{sgn} x)$

$\tanh -1 x, \operatorname{sgn} x \cdot x$

is called exploded $x$ (or exploded of $x$ ) and denoted by $\check{x}$. (Here, $\operatorname{sgn} x=\left\{\begin{array}{c}1, \text { if } x>0 \\ 0, \text { if } x=0 \\ -1, \text { if } x<0\end{array}\right.$,

$\tanh ^{-1} x=\frac{1}{2} \ln \frac{1+x}{1-x},-1<x<1 ;|x|=$

$\int x$, if $x>0$

$\{0$, if $x=0 ;[x]$ is the greatest integer number, $-x$, if $x<0$

which is less than or equal to $x$ and $\{x\}=x-[x]$.)

Moreover, we mention $\breve{x}$ as an exploded number.

Theorem of unabiguouty. (See [1], Theorem 3.2.6.) The mapping $x \leftrightarrow \check{x}$ is mutually unambiguous.

Ordering of exploded numbers: For any pair $x, y \in \mathbb{R}$ we say, that $\check{x}<\check{y}$ if

$$
\begin{gathered}
(\operatorname{sgn} x) \cdot[|x|]<(\operatorname{sgn} x) \cdot[|y|] \\
\text { or } \\
\text { if }(\operatorname{sgn} x) \cdot[|x|]=(\operatorname{sgn} x) \cdot[|y|] \operatorname{then}(\operatorname{sgn} x) \\
\tanh ^{-1}\{|x|\}<(\operatorname{sgn} x) \cdot \tanh ^{-1}\{|y|\}
\end{gathered}
$$

is fulfilled.

Theorem of ordering. (See [1], Theorem 3.2.10. ) For any pair $x, y \in \mathbb{R}, \check{x}<\breve{y}$ if and only if $x<y$.

We say that the points $u=(\xi, \eta) \in \mathbb{R}^{2}$ form a „flag" (in the rectangular Descartes coordinate system of $\mathbb{R}^{2}$ ) if $\eta \in \mathbb{Z}$ and $\xi \cdot \eta \geq 0$. By $(0.1)$ it is clear that the exploded numbers are situated on the „flag”. On the other hand, we have the following

Theorem of completeness. (See [1], Theorem 3.2.8.) If $u=(\xi, \eta) \in \mathbb{R}^{2}$ is situated on the "flag", then

$$
(\eta+\overline{\tanh } \xi)=u \quad, \quad \underset{\xi<\infty)}{(\tanh \xi}=\frac{e^{\xi}-e^{-\xi}}{e^{\xi}+e^{-\xi}},-\infty<
$$

By Theorem of completeness we can say that the „flag" is a geometrical representation of the set of exploded numbers. So, $\widetilde{\mathbb{R}}=\{u=(\xi, \eta) \in$ $\mathbb{R} 2 \xi, \eta=x, x \in \mathbb{R}$. Moreover, we give the compressed of $u \in \widetilde{\mathbb{R}}$, as follows $\underline{u}=\eta+\tanh \xi$, where $u=(\xi, \eta) \in \widetilde{\mathbb{R}}$. Hence, we have that $\underline{u} \in \mathbb{R}$.

Identification. If $u$ is a real number then it is identified with the pair $(u, 0)$, that is $u=(u, 0)$.

\section{SUPER OPERATIONS IN THE SET OF EXPLODED NUMBERS}

The concepts of super - operations. Let $x$ and $y$ be arbitrary real numbers. The super - sum and super - product of $\breve{x}$ and $\breve{y}$ are

and

$$
\check{x} \bar{\oplus} \check{y}=(\overline{x+y}) \quad, \quad x, y \in \mathbb{R} \text {. }
$$

respectively.

$$
\check{x} \bar{\odot} \check{y}=(\overline{x \cdot y}) \quad, \quad x, y \in \mathbb{R},
$$

The mutually and unamgiguous map $x \leftrightarrow \check{x}$ is an isomorphism between the fields $(\mathbb{R},+, \cdot)$ and $(\widetilde{\mathbb{R}}, \bar{\oplus}, \bar{\odot})$.

The monotonity of super - addition. Let $\check{x}, \check{y}$ and $\check{z}$ be arbitrary exploded numbers. If $\check{x}<\check{y}$ then $\check{x} \bar{\oplus} \check{z}<\check{y} \bar{\oplus} \check{z}$ holds. 
The monotonity of super - multiplication. Let $\check{x}, \breve{y}$ be arbitrary and $\check{z}$ be an arbitrary positive exploded numbers. . If $\check{x}<\check{y}$ is valid then $\check{x} \bar{\odot} \check{z}<$ $\check{y} \bar{\odot} \check{z}$ holds.

So, we can prove see that $(\mathbb{R},+, \cdot,<)$ and $(\widetilde{\mathbb{R}}, \bar{\oplus}, \bar{\odot},<)$ are isomorphic ordered fields.

\section{A MATHEMATICAL MODEL FOR THE MULTIVERSE AND PARALLEL UNIVERSES}

In this part the space $\mathbb{R}^{3}$ will be mentioned as ,our universe". Using the explosion of numbers we are able to explode the universe $\mathbb{R}^{3}$. So, the Multiverse

$$
\widetilde{\mathbb{R}^{3}}=\{\check{P}=(\check{x}, \check{y}, \check{z}) \mid P=
$$

$x, y, z \in \mathbb{R} 3$

is obtained. By Theoem of unambiguiuty we can prove that the mapping $P \leftrightarrow \check{P}$ is mutually unambiguous. In general, the point $\check{P}=(\check{x}, \check{y}, \check{z})$ is invisible in our Universe $\mathbb{R}^{3}$. Compressing the $\mathbb{R}^{3}$ we get the open cube

$$
\underline{\mathbb{R}^{3}}=\left\{(\xi, \eta, \zeta) \in \mathbb{R}^{3} \mid \begin{array}{r}
-1<\xi<1 \\
-1<\eta<1 \\
-1<\zeta<1
\end{array}\right\}
$$

It is easy to see that condition $\breve{P}=(\check{x}, \check{y}, \check{z}) \in \mathbb{R}^{3}$ is fulfilled if and only if $P=(x, y, z) \in \underline{\mathbb{R}^{3}}$.

Our first investigated example is the plane $\mathbb{S}=\{P=(x, y, z) \mid z=x+y \quad, x, y \in \mathbb{R}\} . \quad$ Now $\mathbb{S} \cap \underline{\mathbb{R}^{3}}$

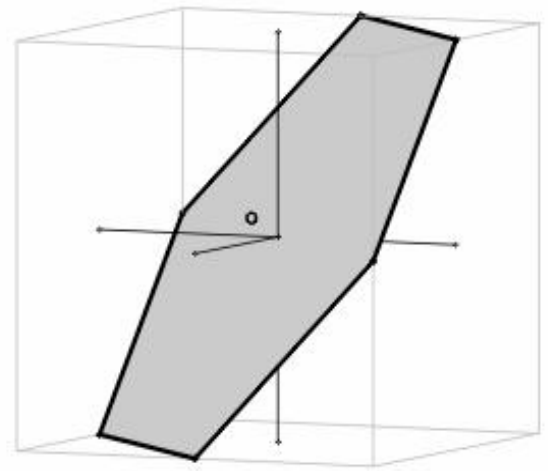

Fig.2.2

is obtained. The exploded of plane $\mathbb{S}$ is the superplane $\quad \breve{S}=\{\check{P}=(\check{x}, \check{y}, \check{z}) \mid P=(x, y, z) \in \mathbb{S}\}$. Exploding the open hexagon presented by Fig. 2.2, we have the visible part of $\mathbb{S}$ $\breve{\mathbb{S}} \cap \mathbb{R}^{3}=\left\{\check{P}=(\check{x}, \check{y}, \check{z}) \mid P=(x, y, z) \in\left(\mathbb{S} \cap \underline{\mathbb{R}^{3}}\right)\right\}$. It is an unbounded set in our Universe.

$$
\widetilde{S} \cap \mathbb{R}^{3}
$$

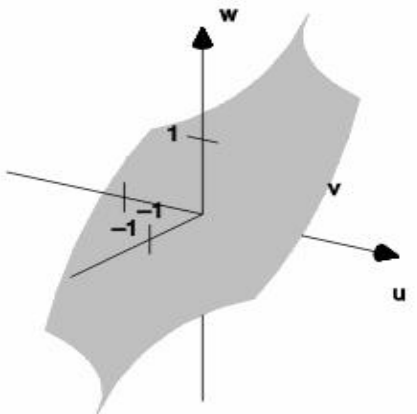

Fig.2.3

(See, [1], point 6.5.) To see the other parts of multiverse $\overline{\mathbb{R}^{3}}$, we use its box - model representation. First of all, we devide the universe $\mathbb{R}^{3}$ by cube-compositions

(2.4) $C_{(p, q, r)}^{b o x}=\left\{(x, y, z) \in \mathbb{R}^{3}|p \leq| x \mid<p+\right.$ $1 ; q \leq y<q+1 ; r \leq z<r+1 ; p, q, r \in \mathbb{N} 0+$

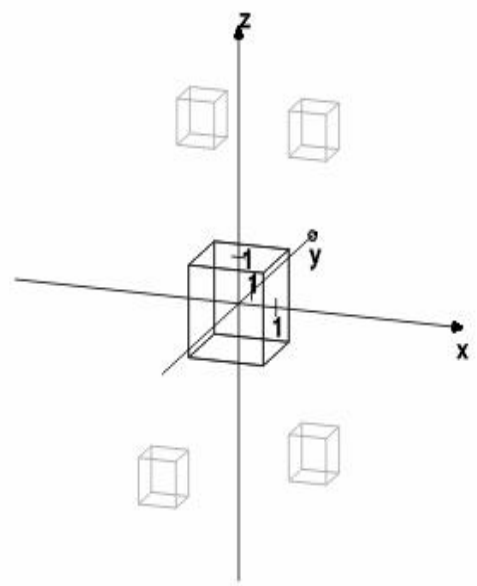

Fig. 2.5

Clearly, $\bigcup_{p, q, r \in \mathbb{N}_{0}^{+}} C_{(p, q, r)}^{b o x}=\mathbb{R}^{3}$ and $C_{(0,0,0)}^{b o x}=\underline{\mathbb{R}^{3}}$. Moreover, each point $P \in \mathbb{R}^{3}$ is situated one and only one cube - composition. Inspired by $(0.1)$ we use the box-exploded of the point $P=(x, y, z) \in$ $\mathbb{R}^{3}$

(2.6) $\breve{P}^{b o x}=\left((\operatorname{sgn} x) \cdot \tanh ^{-1}\{|x|\},(\operatorname{sgn} y) \cdot\right.$ $\tanh -1 y, \operatorname{sgn} z \tanh -1 z, d x, y, z$

where

$$
d_{(x, y, z)}=(\operatorname{sgn} x) \cdot[|x|]+
$$

$(\operatorname{sgn} y) \cdot[|y|] \cdot \sqrt{2}+(\operatorname{sgn} z) \cdot[|z|] \sqrt{3}$.

If $P$ is a point of real number line then $\breve{P}^{\text {box }}=$ $\left((\operatorname{sgn} x) \cdot \tanh ^{-1}\{|x|\}, 0,0,(\operatorname{sgn} x) \cdot[|x|]\right)$

Similarly to the proof of Theorem of unambiguiuty we can prove that the mapping $P \leftrightarrow \breve{P}^{\text {box }}$ is mutually unambiguous. Hence the mapping $\breve{P} \leftrightarrow \breve{P}^{b o x}$ is mutually unambiguous, too. We can say that $\breve{P}^{b o x}$ is a geometric interpretation of $\breve{P}$ in 
the four dimensional space $\mathbb{R}^{4}$. Moreover, for any set $\mathbb{S} \subseteq \mathbb{R}^{3}$

$$
\breve{S}^{b o x}=\left\{\check{P}^{b o x} \mid P=\right.
$$

$x, y, z \in \mathbb{S}$.

Especially, the geometric interpretation of the Multiverse is

$\left\{\breve{P}^{b o x} \mid P=(x, y, z) \in \mathbb{R}^{3}\right\}$.

$$
{\widetilde{\mathbb{R}^{3}}}^{b o x}=
$$

Hence, by (2.6) and (2.9) we can see that ${\overline{\mathbb{R}^{3}}}^{\text {box }} \subset$ $\mathbb{R}^{4}$. By (2.7) we can see that ${\widetilde{\mathbb{R}^{3}}}^{\text {box }}$ is a very thin subset in $\mathbb{R}^{4}$. Using (2.4) - (2.6) we explode the boxes $C_{(p, q, r)}^{b o x}$ and get ,parallel” three - dimensional spaces

$$
\left(\overline{C_{(p, q, r)}^{b o x}}\right)^{b o x}=\{(u, v, w, d) \in
$$

\section{$\mathbb{R} 3$ box}

$-\infty<u<\infty-\infty<v<\infty-\infty<w<\infty d= \pm p \pm q 2 \pm r 3 p$,

(SSCE, $\{(P\}, .6 .2 .12)$ - (6.2.19).) Each point $\breve{P}^{\text {box }} \in$ $\overline{\mathbb{R}}^{3}$ box is situated one and only one three dimensioinal space given by (2.10). Having that $C_{(0,0,0)}^{b o x}=\underline{\mathbb{R}^{3}}$, by (2.6), (2.7) and (2.8)

$$
\begin{aligned}
& \left(\overline{C_{(0,0,0)}^{b o x}}\right)^{b o x}= \\
& \left\{(u, v, w, d) \in{\widetilde{\mathbb{R}^{3}}}^{b o x} \mid \begin{array}{l}
-\infty<u<\infty \\
\begin{array}{l}
-\infty<<<\infty \\
-\infty<w<\infty \\
d=0
\end{array}
\end{array}\right\},
\end{aligned}
$$

is obtained. So, in the box- model in the our universe is represented by $\left(\overline{C_{(0,0,0)}^{b o x}}\right)^{b o x}$.

The investigated plane $\mathbb{S}$ turns out from $C_{(0,0,0)}^{b o x}$ and a part of $\mathbb{S}$ is situated in

$$
\begin{gathered}
C_{(0,0,1)}^{b o x}=\left\{(x, y, z) \in \mathbb{R}^{3} \mid-1<x<1 ;-1<y\right. \\
<1 ; 1 \leq|z|<2\}
\end{gathered}
$$

which is one of the neigbour universes of our universe.

$$
\mathbb{S} \cap \text { Right - Back-Upper part of } C_{(0,0,1)}^{b o x}
$$

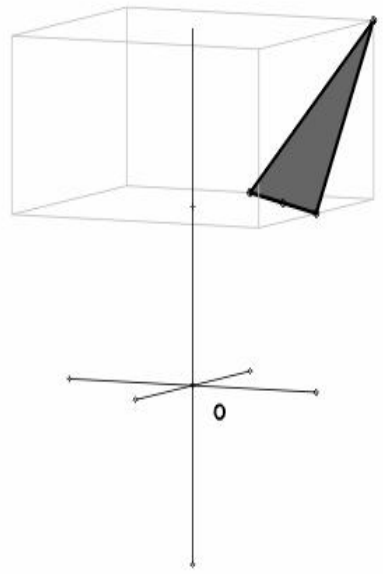

Fig. 2.11
If a point $P=(x, y, z)$ is situated in ,Right - BackUpper" part of the box $C_{(0,0,1)}^{b o x}$, then

$0 \leq x<1,0 \leq y<1$ and $1 \leq z<2$ holds. (See Fig. 2.11) Hence, (2.6) and (2.7) yields $\breve{P}^{b o x}=\left(\tanh ^{-1} x\right.$, $\tanh -1 y, \tanh -1 z-1,3$.

Moreover, by (2.10) the universe $\left(\overline{C_{(0,0,1)}^{\text {box }}}\right)^{\text {box }}=$

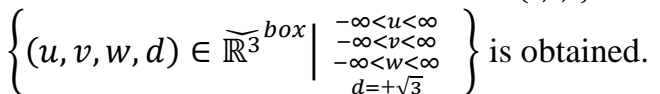

Denoting $u=\tanh ^{-1} x, v=\tanh ^{-1} y$ and $w=\tanh ^{-1}(z-1)$ and having that if $P \in \mathbb{S}$ then $\breve{P}^{b o x} \in \breve{\mathbb{S}}^{b o x}$, by (2.12) and the equation $z=x+y$ we can write

$$
\begin{gathered}
w=\tanh ^{-1}(z-1)=\tanh ^{-1}(x+y-1) \\
=\tanh ^{-1}(\tanh u+\tanh v \\
-1) .
\end{gathered}
$$

So, $\breve{\mathfrak{S}}^{\text {box }} \cap$ upper part of $\left(\overline{C_{(0,0,1)}^{\text {box }}}\right)$ box which is the exploded of $\mathbb{S} \cap U$-part of $C_{(0,0,1)}^{b o x}$ has

(2.13) $\quad w=\tanh ^{-1}(\tanh u+\tanh v-$

$1 \quad, 1 \leq \tanh u+\tanh v<2, u, v \in \mathbb{R} O+$

This is the equation of continuation of $\breve{\mathbb{S}} \cap$ $\left(\overline{C_{(0,0,0)}^{b o x}}\right)^{b o x}$ introduced on Fig. 2.3.

$$
\breve{\mathfrak{S}}^{\text {box }} \cap \text { upper part of }\left(\overline{C_{(0,0,1)}^{b o x}}\right)^{b o x}
$$

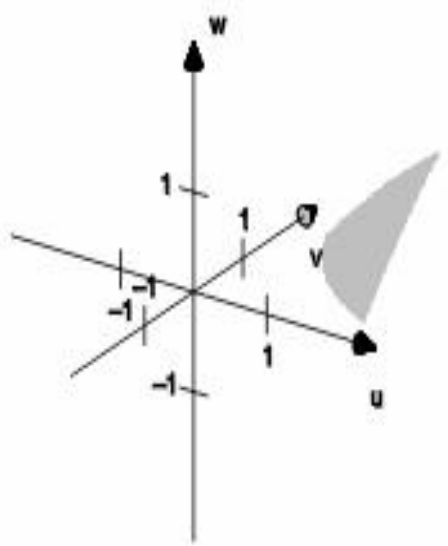

Fig. 2.14

By (2.13), in the ,highness" $w=\breve{1}$ the level - curve of super-plane $\breve{S}$ has the equation $\tanh u+\tanh v=1, u, v \in \mathbb{R}_{0}^{+}$.

This level - curve is situated on the border of our universe, so, it is invisible for us, but it is visible in the ,neighbouring" universe $\left(\overline{C_{(0,0,1)}^{\text {box }}}\right)$ box . (See the „u,v" plane on Fig. 2.14.)

In our second example we imagine that a surveyor starts from the origo of our Universe and moves on the super - line $\widetilde{\mathbb{L}}=\{\breve{P}=(\check{x}, \breve{y}, \check{z}) \mid P=(x, y, z) \in$ $\mathbb{L}$,where 


$$
\mathbb{L}=\{P=
$$

$x, y, z \in \mathbb{R} 3 x=16 \cdot t y=16 \cdot t z=26 \cdot t, t \in \mathbb{R}$

The line $\mathbb{L}$ bores through infinite many box compositions given under (2.4). The points

$$
\begin{gathered}
K=\left(\frac{3}{2}, \frac{3}{2}, 3\right) \quad, \text { see } t=\frac{3 \sqrt{6}}{2}, \\
H=(1,1,2), \text { see } t=\sqrt{6}, \\
G=\left(\frac{1}{2}, \frac{1}{2}, 1\right) \quad, \text { see } t=\frac{\sqrt{6}}{2}
\end{gathered}
$$

and the origo $\mathcal{O}=(0,0,0)$ are denoted on the next figure.

Some passages of the line $\mathbb{L}$

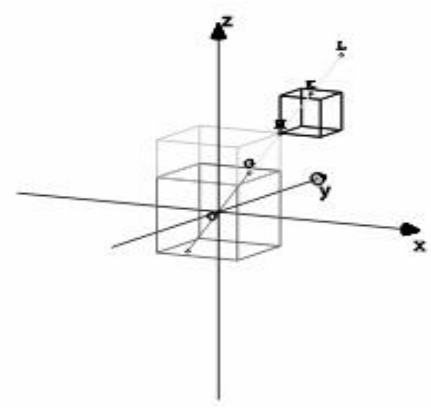

Fig. 2.16

By (2.4) and (2.15) we can see that $[\mathcal{O}, G[=$ $\left\{P=\left.(x, y, z) \in \mathbb{R}^{3}\right|^{\substack{x=\frac{1}{\sqrt{6}} t \\ y=\frac{1}{\sqrt{6}} t}}, 0 \leq t<\frac{\sqrt{6}}{2}\right\}$. Hence, the passage of the the way of the surveyor situated in our Universe

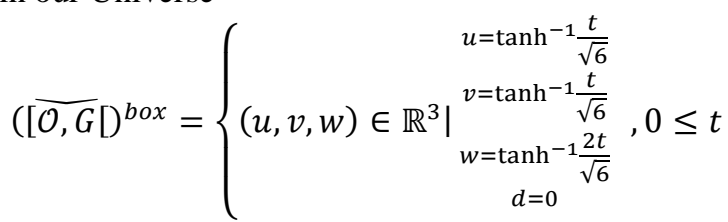

$$
\begin{aligned}
& \left.<\frac{\sqrt{6}}{2}\right\} \\
& \left(\overline{[\mathcal{O}, G}[)^{b o x}\right.
\end{aligned}
$$

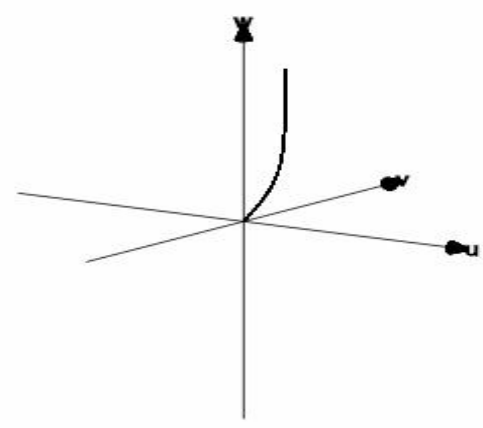

Fig. 2.17

The Fig. 2.17 perceives that $\lim _{t \rightarrow \frac{\sqrt{6}}{2}} w(t)=\infty$. So, $t<\frac{\sqrt{6}}{2}$

$\left(\left[\overline{\mathcal{O}, G}[)^{b o x}\right.\right.$ is unbounded in our Universe. The point $\breve{G}=\left(\widetilde{\left(\frac{1}{2}\right)}, \widetilde{\left(\frac{1}{2}\right)}, \check{1}\right) \notin \mathbb{R}^{3}$ but $\breve{G}^{\text {box }}=$ $\left(\tanh ^{-1} \frac{1}{2}, \tanh ^{-1} \frac{1}{2}, 0, \sqrt{3}\right) \in\left(\overline{C_{(0,0,1)}^{\text {box }}}\right)^{\text {box }}$. The surveyor turns into a new three - dimensional universe $\left(\overline{C_{(0,0,1)}^{b o x}}\right)^{b o x}$ :

$$
\begin{gathered}
\left(\left[\overline{G, H}[)^{b o x}=\left\{\left.(u, v, w) \in \mathbb{R}^{3}\right|_{\begin{array}{c}
w=\tanh ^{-1}\left(\frac{2 t}{\sqrt{6}}-1\right) \\
d=\sqrt{3}
\end{array}} ^{\substack{u=\tanh ^{-1} \frac{t}{\sqrt{6}}\\
}}, \frac{\sqrt{6}}{2}\right.\right.\right. \\
\leq t<\sqrt{6}\} .
\end{gathered}
$$

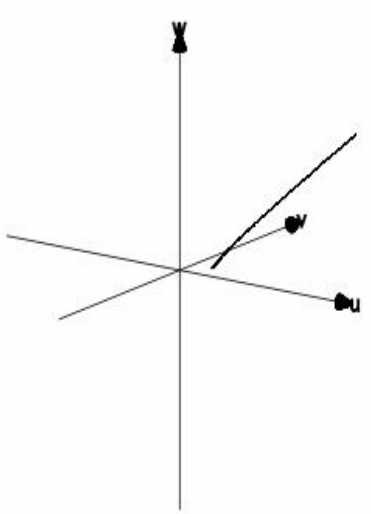

Fig. 2.18

As

$$
\lim _{\substack{t \rightarrow \sqrt{6} \\ t<\sqrt{6}}} u(t)=\infty, \lim _{\substack{t \rightarrow \sqrt{6} \\ t<\sqrt{6}}} v(t)=\infty \text { and } \lim _{\substack{t \rightarrow \sqrt{6} \\ t<\sqrt{6}}} w(t)
$$$$
=\infty \text {, }
$$

the surveyor moves towards the point $\breve{H}=$ $(\breve{1}, \breve{1}, \breve{2})$. The box - explosion formula (2.6) with (2.7) shows that $\breve{H}^{\text {box }}=(0,0,0,1+\sqrt{2}+2 \sqrt{3})$ where the surveyor arrives. It is the origo of the 


$$
\begin{aligned}
& \text { three - dimensional space }\left(\overline{C_{(1,1,2)}^{\text {box }}}\right)^{b o x}= \\
& \left\{(u, v, w, d) \in{\widetilde{\mathbb{R}^{3}}}^{b o x} \mid \begin{array}{c}
-\infty<u<\infty \\
-\infty<<<\infty \\
-\infty<w<\infty \\
d= \pm 1+ \pm \sqrt{2} \pm 2 \sqrt{3}
\end{array}\right\} \text {. By } \\
& \left(\left[\overline{H, K}[)^{b o x}=\{(u, v, w)\right.\right.
\end{aligned}
$$

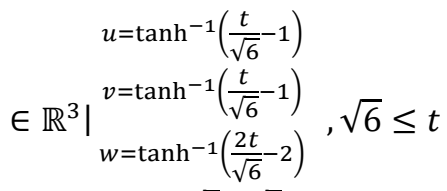

$$
\begin{aligned}
& \left.<\frac{3 \sqrt{6}}{2}\right\}^{d=1+\sqrt{2}+2 \sqrt{3}}
\end{aligned}
$$

and the substitution $\tau=t-\sqrt{6}, \sqrt{6} \leq t<\frac{3 \sqrt{6}}{2}$ we have the Fig. 2.17, again. The surveyor tends to the point $\breve{K}=\left(\overline{\left(\frac{3}{2}\right)}, \overline{\left(\frac{3}{2}\right)}, \breve{3}\right)$ assuming that it has sufficient capacity to move.

Fig. 2.17 and 2.18 show that the further way in the multiverse is periodical.

\section{ACKNOWLEDGEMENT}

Thanks for support of the Foundation of Szeged.

\section{REFERENCE}

[1]. István Szalay: Exploded and compressed numbers, (Enlargement of the universe, Parallel Universes, Extra Geometry), LAMBERT Academic Publishing, Saarbrücken, Germany, 2016, ISBN: 9783-659-94402-4.

Szalay, I. "A mathematical model of the Multiverse described by exploded numbers." International Journal of Engineering Research and Applications (IJERA) 7.8 (2017):25-29 